\title{
ZUF̈̈LLIG GUT? ÜBER LIVE-PERFORMANCES UND VIRTUOSITÄTSPOTENTIALE. Helene Fischers Berliner Auftritt im Regen
}

\author{
Barbara Hornberger und Christoph Jacke
}

\section{Einleitung}

»Miele, Miele, sprach die Tante, die alle Waschmaschinen kannte« (Miele, 1926) oder »Aus Erfahrung gut (AEG, 1958 u. 1976) - Werbe-Slogans setzen auf Vertrauen: Erfahrung, das bedeutet abgesichertes Wissen, fundierte Kenntnisse, Verzicht auf waghalsige Experimente. Wer das kauft, so die Botschaft, der geht kein Risiko ein, der kauft keine Katze im Sack, der erwirbt ein ausgereiftes, erprobtes Produkt, egal, ob es sich um eine Waschmaschine, einen Staubsauger oder einen Föhn handelt. Denn niemand möchte etwas kaufen, das nur zufällig gut ist, bei dem man Glück haben muss, wenn es hält, was man sich davon verspricht - so wie die Persiflage des Slogans »AEG - Aufstellen - Einschalten - Geht nicht « von der Nicht-Einhaltung des Funktions-Versprechens erzählt. Heute werden die von den Slogans behaupteten Lebens- und somit auch Waschmaschinen-Erfahrungen der Tante im Netz durch Bewertungen und Testergebnisse ergänzt, die die vollmundigen Werbeversprechungen der Hersteller durch sechte- Erfahrungswerte anderer Käufer und Nutzer verifizieren. Der Bedarf nach dieser Form von Vertrauensbildung ist da, denn die Warenwelt ist eine, in der ausgewählt wird, und Verlässlichkeit ist in diesem Prozess von immenser Bedeutung. Diese Art von Vertrauen und die damit verbundenen professionalisierten Vertrautheitsinszenierungen (vgl. Zurstiege 2005: 198) nutzen wir auch für kulturelle Waren. Genres z.B. sind von der Kulturindustrie etablierte, bedeutende Komplexitäts- und somit Risikoreduzierer, die uns die Auswahl erleichtern, weil wir uns darauf verlassen, dass CD-Cover, Filmplakate oder auch nur Casts Hinweise darauf geben, was wir zu erwarten haben. Und noch eindeutiger fällt die Wahl aus, wenn wir bereits eigene Erfahrungen mit einem 
Produkt haben, die die Tante überflüssig machen können: Denn so wie wir genau wissen, wie der Cheeseburger in der Burgerkette schmecken wird, wissen wir auch, was wir von Wacken zu erwarten haben, von einem Bruce Springsteen-Konzert oder einem Helene Fischer-Abend. Wobei der Unterschied sein dürfte, dass wir bei den Cheeseburgern den genormten, immer gleichen Geschmack in Kauf nehmen - vielleicht sogar schätzen -, während wir bei Wacken, Springsteen oder Fischer durchaus in jeder Performance Variationen erkennen (wollen).

Auf je eigene Weise ist jedes dieser Konzerte kultürlich eine genau abgestimmte Inszenierung, in der die Live-Präsentation der Songs verknüpft wird mit der performativen Darstellung des Star-Images und einem Gemeinschaftserlebnis von Star und Publikum. Dabei ist von entscheidender Bedeutung, dass die Inszenierung die Erwartung des Publikums in irgendeiner Form trifft und dieses im Sinne Simon Friths (1996) zum Bestandteil der Performance wird: Auch ein einfaches »Nur-auf-der Bühne-Sein« folgt dieser Logik und unterstützt - z.B. bei Nirvana - das entsprechende Image (hier: Verweigerung von Glamour, Anti-Showbiz).

Bei Live-Inszenierungen populärer Musik lassen sich verschiedene Dimensionen von Virtuosität beobachten und unterscheiden, die alle gleichermaßen die Ereignishaftigkeit der Aufführung und das jeweilige Star-Image unterstützen, die sogar - als in Aussicht gestellte Leistung - Konsumentscheidungen wie den Kauf von Eintrittskarten attraktiv machen. In Live-Situationen wird zudem der Zufall als Mitspieler interessant. Denn in vom Zufall beeinflussten Situationen entfaltet sich die Virtuosität des Performativen, die wir besonders in den Blick nehmen und am Beispiel eines Helene Fischer-Konzerts explorieren wollen.

\section{Inszenierung und Aufführung: Zufällige Performances - performte Zufälle}

Live-Konzerte sind präzise, auf Erwartungshorizonte und Imagetransfers angelegte Inszenierungen. Als Inszenierungen bezeichnen wir in diesem Zusammenhang den »Vorgang der Planung, Erprobung und Festlegung von Strategien [...], nach denen die Materialität einer Aufführung performativ hervorgebracht werden soll« (Fischer-Lichte 2005b: 146). Inszenierungen bedürfen also des Moments der Aufführung, um ihre Wirkung zu entfalten. ${ }^{1}$

1 Diese Definition des Inszenierungs- und Aufführungsbegriffs ist abzugrenzen von erweiterten Konzepten von Performance und Liveness im digitalen Zeitalter, wie sie etwa Philip Auslander (2012) entwickelt hat. In Fischer-Lichtes theater- 
Mit Richard Schechner (1988: 72) würde man vom »Script « sprechen als »the basic code of the event «. Als Aufführung - oder mit Schechner »Performance - gilt das jeweils einzigartige und gegenwärtige Ereignis, das vor und mit Publikum realisiert wird. Zuschauer und Zuschauerinnen und ihre »leibliche Ko-Präsenz « (Fischer-Lichte 2005a: 16) mit den Stars im Handlungsraum Konzert sind daher für die Aufführung als Ereignis konstitutiv. Ob dieses Ereignis als > gut < oder eben >nicht gut < beurteilt wird, hängt maßgeblich von der Passgenauigkeit der Inszenierung in Bezug auf das Star-Image ab, aber eben auch davon, was in der jeweilig einmaligen Aufführung, in der Interaktion mit dem Publikum, im transitorischen und flüchtigen Geschehen des Konzerts, wirklich geschieht, und zwar auf der Ebene der Vergemeinschaftung wie auf der Ebene der Star-Performance. In den meisten Popkonzerten finden sich Elemente, die das Zusammenspiel und das Gemeinschaftserlebnis, aber auch die Ereignishaftigkeit und Einzigartigkeit verstärken. Solche Elemente integrieren das Publikum als handelnd in den Ablauf. Das können einfache Kommunikationsakte sein (schon das Kasperle ruft: »Seid Ihr alle da« und das Publikum ruft »Ja« zurück), musikalische Interaktionen (Mitsingen, Call and Response, Klatschen), und performative Handlungen (Stage Diving, Air Guitar spielen). Die Beteiligung des Publikums ist also erwünscht, ja, notwendig. Zugleich wirkt das Publikum wie ein Zufalls-Generator, weil sich seine Handlungen nicht im Detail kalkulieren lassen, und das kann, insbesondere für choreografisch und szenografisch besonders aufwändige Inszenierungen, zum Problem werden. In vielen Shows wird darum im Voraus geplant, dass und in welchem Rahmen Publikumshandlungen evoziert werden. Dafür werden inszenatorische Pre-Sets eingesetzt, die die Spontaneität und damit auch den Zufall kalkulierbar machen.

Allerdings geht es eben im Live-Konzert auch um das Hervorbringen, Zeigen und Bestätigen der Star-Figur, die auf der Folie des bereits medial entstandenen Images performt und rezipiert wird. Und dieser Vorgang ist mit der Inszenierung eng verknüpft, denn von ihr hängt ab, wie diese Performance gestaltet werden kann. Eine vor allem von spektakulären Effekten geprägte Show zeigt Stars - wie etwa Madonna, Michael Jackson, Lady Gaga, Rammstein oder auch in den 1970er Jahren Pink Floyd - als Teil eines gesamtkünstlerischen Panoramas, ein (scheinbar!) wenig inszeniertes Büh-

wissenschaftlichem Aufführungsbegriff ist die leibliche Ko-Präsenz beim Erleben des in dieser Situation unberechenbaren Zufallseinbruchs elementar. Da wir in unserem Beitrag vorrangig Live-Situationen in den Blick nehmen, scheint uns diese Unterscheidung sinnvoll und ihre Bedingung angemessen. Sobald LiveEreignisse analog oder digital reproduzierbar werden und in Serie gehen oder Anwesenheit virtuell wird, müsste unser Konzept, z.B. mit Auslander, modifiziert werden. 
nengeschehen kann wiederum Nähe zum Fan und die >Natürlichkeit des Stars vermitteln. In jedem Fall gibt die Inszenierung die Rahmenbedingungen nicht nur für das Agieren des Publikums vor, sondern auch für die Stars und ihre Hervorbringung der Bühnen- und Starfigur in ihrem künstlerischen und performativen Handeln. Um dieser Performance zusätzlichen Raum zur Entfaltung zu geben, stellen manche Popkonzerte innerhalb der geplanten Inszenierung Freiräume, Leerstellen bereit, in denen nicht vollständige kalkulierbare Interaktionen stattfinden können. Wenn - wie z.B. beim Robbie Williams-Konzert in Knebworth (England) im Jahr 2003 - einzelne Fans auf die Bühne geholt werden, wenn längere Gespräche mit ausgesuchten Personen aus dem Publikum geführt werden, ist die Situation offener; der Verlauf hängt dann von beiden - Star und Fan - ab. Dabei begegnen sich auch Medienprofis und Bühnen-Laien, sofern diese Situationen nicht auch durchgeplant und -choreographiert sind. Und je ungewöhnlicher die Situation verläuft (häufig durch eine nicht erwartete Handlung der beteiligten >Laien<), desto einzigartiger und nicht-konventioneller wirkt sie und desto mehr hat der Star die Möglichkeit, sein Image respektive seine Bühnenfigur performativ zu zeigen (vgl. Hornberger 2017). Durch den gelenkten Zufall (vgl. Großmann 1999: 125-129) entsteht ein spezifischer, aleatorisch geprägter Handlungsraum, in dem sich der Star beweisen kann - aber auch muss. Es hängt maßgeblich von der Souveränität des Stars ab, ob solche Situationen gelingen und etwas Besonderes hervorbringen, ob sie sgut s sind - und in diesem Sinn bedeutet > gut< zumeist als Zuschreibung innerhalb von Authentifizierungsstrategien glaubwürdig und >un-inszeniert < (vgl. Jacke 2013).

Wie offen solche Inszenierungen gehalten werden können, hängt auch davon $a b$, wie souverän der Star das Spiel mit dem eigenen Image beherrscht. Das Image des Popstars kann dort besonders hervortreten, wo die Inszenierung über die reine Song-Aufführung hinausreicht, darum werden solche Momente vor allem bei den Konzert-Inszenierungen von Stars eingebaut, die mit ihrer Bühnenfigur und ihrem Image sicher, also spielerisch umgehen können. ${ }^{2}$ Der gelenkte Zufall im Sinne Großmanns ermöglicht dem Star das Ausspielen seiner Performer-Qualität und bietet im Fall des Gelingens dem Publikum ein spezifisches ästhetisches und soziales Erlebnis: »Die Künste wollen den Zufall, benötigen ihn geradezu systematisch. Sie versuchen ihn eben deshalb auf immer neue Weise zu platzieren,

2 Wir verstehen Images hier als im weiten Sinne multimediale Gesamt-Texte (vgl. Jacke 2011), die von den historische Personen, den beteiligten Medien und den Rezipienten gleichermaßen geschaffen werden (vgl. Lowry 2003; Hügel 2007). 
[...] herzustellen, an einen wichtigen Punkt im ästhetischen Gefüge zu stel-

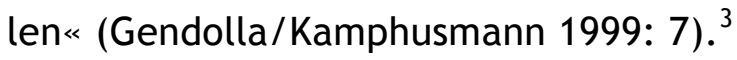

\section{Virtuositätsbegriffe populärer Musik}

Es lassen sich bis hierhin im Kontext Live-Konzert zunächst zwei verschiedene Formen von Virtuosität unterscheiden, die jeweils mit der Inszenierung und ihrem Umgang mit Zufall eng zusammenhängen. Eine genau geplante Konzert-Inszenierung zeigt Virtuosität eher auf der Ebene der Show als Ganzes - im Ineinandergreifen von visuellen Effekten, Licht-Technik, Sound, Choreografie und der sich darin souverän bewegenden Künstler. Virtuosität ist hier sowohl seitens der Regie als auch seitens der Technik und natürlich auch seitens der Ausführenden notwendig, damit das Spektakuläre seine überwältigende Wirkung entfalten kann. In den Inszenierungen, die Momente der Unkalkulierbarkeit zur Verfügung stellen, liegt wiederum die Virtuosität deutlicher bei den performenden Musikerinnen und Musikern, in der souveränen Beherrschung der eigenen Bühnenfigur sowie der Situation, die durch den Zufall bestimmt ist. Wer sich hier beweist, kann seine Star-Figur nicht nur zeigen, sondern zugleich authentifizieren.

Zunächst sind Virtuosität und der Virtuose als »eine Figur der (Über-) Steigerung « beschreibbar, die »in einem bestimmten Typ von Performer « inkorporiert ist, als ein Konzept, das vom 17. Jahrhundert bis in die Gegenwart »im Kontext abendländischer Kulturen Grenzen des menschlichen Handelns angeht, sie im Zuge ihrer Achtung und Missachtung, ihrer Überschreitung, Um- oder Überspielung evident macht und den Wert dieser Evidenz in künstlerische, ökonomische oder politische Kalküle integriert « (Brandstetter/Brandl-Risi/Eikels 2017: 9). Für diesen Vorgang sind, wenn er im Bereich des Künstlerischen stattfindet, ${ }^{4}$ künstlerische und performative Fähigkeiten

3 Bisher wenig behandelt scheint der Begriff des Zufalls in den Kultur-, Medienund Musikwissenschaft und erst Recht in der Popmusikkulturforschung. Hier sind die Beiträge in dem von Peter Gendolla und Thomas Kamphusmann herausgegebenen interdisziplinären Sammelband Die Künste des Zufalls (1999) hilfreich, insbesondere sowohl die eher naturwissenschaftlichen Einführungstexte von Claus Grupen und Wolfgang Coy als auch die eher auf Musik und in Ansätzen sogar das Populäre bezogenen Überlegungen von Holger Schulze und Rolf Großmann.

4 Ob die Kategorie des Virtuosen auch für ökonomische und politische Felder in Anspruch genommen werden kann, ist durchaus umstritten, Denn häufig werden diese im Diskurs dem Bereich der Ästhetik entgegengesetzt. Dies gilt implizit schon, wenn etwa Hegel den 'wahren< (nämlich schöpferischen) vom sbloßen< (lediglich handwerklich-technischen) Virtuosen unterscheidet (vgl. Brandstet- 
zugleich notwendig: Ein Virtuose kann charakterisiert werden als ein Künstler, »der etwas kann und dies auf exponierte Weise zeigt « (Hügel 2003: 491), womit er andere übertrifft. Im 18. und 19. Jahrhundert ist das Virtuose in erster Linie eine Domäne der Musik, ${ }^{5}$ im Kontext des bürgerlichen Konzertlebens werden unter dem gezeigten >Können< üblicherweise künstlerisch-handwerkliche Fähigkeiten verstanden, z.B. eine besondere Fingerfertigkeit oder Stimmgewalt. Aus diesem Grund sind Virtuosen oder Virtuosinnen stets professionelle Künstler und als solche Teil des öffentlichen Kulturbetriebs. Aus dieser Verortung heraus entsteht die

»Überzeugung, der Ursprung von Begriff und Konzept des Virtuosen liege in der Kunst; die Unterstellung einer Deckungsgleichheit von Kunst und >dem Ästhetischen<; und vor allem die Annahme, Kunst, Ökonomie und Politik seien zwar prozessual irgendwie verflochten, wertemäßig aber sauber voneinander abgrenzbare Bereiche mit ihren je eigenen Kriterien und einem spezifisch zuständigen Vokabular, das die Wertseparation bewacht« (Brandstetter/Brandl-Risi/Eikels 2017: 8).

Hierin sehen Brandstetter et al. »Effekte einer Fraglosigkeit, die stille Abmachungen in der Allianz von bürgerlicher Kultur und akademischen Kultur-Wissenschaften schützt« (ebd.). In dieser Überbetonung des Künstlerischen gerät jedoch aus dem Blick, dass das öffentliche Aufführen der herausragenden Fähigkeiten dem Publikum das Verstehen der Leistung und den Genuss erheblich erleichtert, gerade in der Musik: Denn es muss die herausragende Leistung nicht hörend erkennen, sie wird ihm außerdem gezeigt. So verstanden ist Virtuosität bereits ein Popularisierungsvorgang (vgl. Hügel 2003).

ter/Brandl-Risi/Eikels 2017: 16f.). Was nicht schöpferisch ist, ist demnach auch nicht wirklich virtuos, schlimmer noch: Das nur scheinbar Virtuose ist suspekt: »Die Instrumentalisierung der ästhetischen Dignität von Kunst für irreguläre Gewinne, die ihren ökonomisch und politisch interessierten Charakter durchblicken lassen, kommt den Spekulationen auf eine gute, gesellschaftlich bildende Kraft ästhetischer Autonomie in die Quere. Die spielerische Freiheit des Virtuosen droht vorab das zu vergiften, was den zum Publikum versammelten Subjekten ästhetischer Erfahrung von autonomer Kunst an Befreiung geschenkt werden soll. [...] Die heute vorherrschende Verengung des Verständnisses von Virtuosität auf >die Kunst<, gleichgesetzt mit >dem Ästhetischen<, vergisst diese Konflikte, die bis in die Entstehungsphase der ästhetischen Theorie zurückdatieren« (ebd.: 17).

5 Damit zentral verbunden ist die Bühne als der Ort, an dem diese Virtuosität gezeigt und bezeugt wird. Nicht zufällig gelten daher auch einzelne Tänzerinnen wie Marie Taglioni und Fanny Elßler und etwas später Schauspieler und Schauspielerinnen wie Frédérick Lemaître, Sarah Bernhardt oder Eleonora Duse als virtuos. 
Diese Ambivalenz ist bis heute unaufgelöst: Auch in der populären Musik wird Virtuosität (insbesondere das Gitarrensolo) als eine an der E-Musik orientierte Leistungsschau und ostentative Dauer-Überbietung zugleich gefeiert und kritisiert. In der Betonung der künstlerischen Leistung gerät der Blick auf die performative Leistung häufig flüchtig, sie wird mitunter sogar als effekt- und affekthaschend und übertrieben und daher störend wahrgenommen oder sogar relativiert, ironisiert und lächerlich gemacht. Gerade in der populären Musik gilt noch immer weitgehend, was Peter Wicke konstatierte: die »Abwesenheit des Begriffs >Virtuosität« (Wicke 2004: 232) in der Begriffswelt der Popmusik und ihrer Erforschung. Dies lässt sich zum einen auf die Verortung des Begriffs in der bürgerlichen E-Kultur zurückführen, die tendenziell noch immer als eine gilt, gegen das Populäre sich abzugrenzen habe. Zum anderen aber liegt das Problem der mangelnden Erforschung auch darin, dass das

»Urteil >virtuos!< [...] selbst genuin unsachlich [ist]. Es enthält einen Exzess an Wertzuschreibung, ein Zuviel, das es zugleich als Akt der Anerkennung fragwürdig macht. Es lässt sich weder phänomenal objektivieren noch in einer allgemein nachvollziehbaren Evaluation verankern« (Brandstetter/ Brandl-Risi/Eikels 2017: 10).

Es erscheint daher zunächst einmal naheliegend, den Begriff weiter ruhen zulassen. Allerdings kommen mit dem Begriff der Virtuosität dann doch bestimmte Aspekte von Popmusik in den Blick, die bei aller Unschärfe für die Texte und Kontexte von Popmusik (und freilich die Diskussionen darum) von erheblicher Bedeutung sind: das Spektakuläre, die Frage nach Leistung und ihrer subjektiven oder objektiven Bewertung, die emotionale Qualität von Live-Performances. Diese Dimensionen lassen sich, wie wir meinen, über den Begriff der Virtuosität auch in ihrer Verbindung beobachten und erschließen. ${ }^{6}$

Tatsächlich erläutert bereits Wicke profund, dass Konzepte von Virtuosität nur scheinbar unpassend für die Reflexion und Erforschung von Popmusik sind. Wicke unterscheidet die musikalisch-ästhetische, sozial-kulturelle und symbolische Dimension von Virtuosität und betont die Bedeutung der popmusikalischen Aufführung und der Performances ihrer Akteure:

»[E]ntscheidend ist der Charakter der Einmaligkeit und nach Möglichkeit auch des Scheins der Unwiederholbarkeit, der die Performance als Form der

6 Zu dem Themengebiet scheinen die beiden grundlegenden Beiträge zur musikalischen Virtuosität von Wicke (2004) und Hanns-Werner Heister (2004) weiterhin ebenso angebracht wie Hans-Otto Hügels Ausführungen zum Virtuosen (2003). 
Begegnung von Musiker und Publikum zumindest temporär den Apparaturen der Musikindustrie entzieht« (Wicke 2004: 236).

Vor dem Hintergrund von Performativität lassen sich die drei von Wicke genannten Dimensionen modifizieren: Virtuosität populärer Musik verlangt in ihrer Aufführung Kompetenzen, die mal eher technisch ausgefeilt scheinen (musikalisch-technisch), mal in einem besonderen Auftreten (sozial-kommunikativ) liegen, oder in dem Gespür für die Situation und ihre Gestaltung (situativ-performativ) liegen. ${ }^{7}$ Die Verbindung von auditiver und visueller Wahrnehmung von Virtuosität betonen auch Hans-Otto Hügel (2003) und Simon Frith (1996), indem sie Sinnlichkeit und Körperlichkeit als zentrale Faktoren populärer Musik akzentuieren: »[T]o hear music is to see it performed, on stage, with all the trappings « (Frith 1996: 211). Dies hat auch grundsätzliche Konsequenzen für die Konzeptualisierung von populärer Musik: »In den populären Musikformen steht nicht das >Werk im Mittelpunkt, auch nicht in Analogie dazu der Song, sondern vielmehr die >Performance « (Wicke 2004: 234).

Erst im Gegenüber aus Performenden und Zuschauenden entsteht letztlich sozial-kommunikativ eine Kooperation, ein Gemeinsames und sei es noch so para-interaktiv. In »Musik mit ihrer komplexen inneren Dialektik von Ich und Wir, Individualität und Kollektivität, werden die beiden Grundformen menschlicher Beziehungen - Kooperation und Konfrontation - auf besondere Weise gespiegelt « (Heister 2004: 19-20). Es geht dabei oft mehr um die »Komplizenschaft « (Wicke 2004: 235) zwischen Star und Fan als um das meisterhafte Beherrschen der Musik-Interpretation oder gar der Instrumente. Doch auch wenn sich die Aufmerksamkeit auf die Bühne und die Performance verlagert, so scheint eine gewisse Mühelosigkeit des Auftritts doch wichtig, um den Eindruck von Virtuosität hervorzurufen. Heister spricht hier vom speziellen »Als ob«, einem Effekt, der alles quasi automatisch gelingend erscheinen lässt, und nennt dies das »Zirkus-Prinzip«, wobei das Gelingen - als Zeigen von Fähigkeiten - wichtiger ist als die Qualität der Herausforderung. »Bei Virtuosität ist überdies mindestens zu differenzieren zwischen der Meisterung tatsächlicher Schwierigkeiten und der Darstellung zwar effektvoller, aber bloß scheinbarer technischer Schwierigkeiten« (Heister 2004: 24). Das »Als ob« kann nach Frith durchaus naturalisiert und

7 »Der Virtuose sollte zwar ein Interpret von höchstem Rang sein, aber auch ein Konzertereignis « (Motte-Haber 2004: 179). An H. Kuppens und Frank van der Pol (2014) haben für niederländische Black Metal-Fans konstatiert, dass Authentizität innerhalb der Musikindustrie von Konsumenten gleichbedeutend bewertet wird wie die musikalischen Fähigkeiten eines Künstlers und somit zum integralen Bestandteil der kommunikativen Erfahrung eines Konzert-Ereignisses werden kann. 
in die Performance integriert sein: "It's as if the sas if < of the song performance is foregrounded in order to naturalize the sas if < of the musical performance « (Frith 1996: 211). In jedem Fall geht es darum, Herausforderungen - des Instruments, des Werks, der Situation - zu meistern, das Virtuose entsteht in einem vielfältigen Dazwischen:

»Der Virtuose steht daher historisch und systematisch zwischen dem Künstler, der den Status eines Handwerkers hat, und dem, der ein Star ist. Kommt es bei jenen nur auf die Leistung an, die er hervorbringt, geht es bei diesem vor allem um das von ihm hergestellte Image. Wird etwa bei einem höfischen Tafelmusiker vor allem das dargebotene Musikstück rezipiert, kommuniziert man beim Star mit der Kunst-Figur, die dialektisch Selbst- und Rollendarstellung verbindet« (Hügel 2003: 492).

Diese Mehrdimensionalität kann soweit führen, dass sogar vermeintlicher Dilettantismus hoch organisiert und virtuos >gemeistert < wird, wie etwa bei Helge Schneider, oder dieser bewusst zelebriert, zur Kunstform erklärt wird wie bei den Musikerinnen und Musikern der Genialen Dilettanten. ${ }^{8}$

\section{Helene Fischer live oder: Der Einbruch des Regens}

Live-Konzerte verlangen Virtuosität in allen drei von uns genannten Dimensionen: Selbstverständlich sollen Künstlerinnen und Künstler zeigen, dass sie ihre Stimme oder ihr Instrument musikalisch-technisch beherrschen, und genau so wird erwartet, dass sie ihr Star-Image kommunikativ hervorbringen und bestätigen. Situativ-performative Virtuosität ist der transitorischen Qualität der Aufführung ohnehin eingeschrieben, sie ist allerdings in besonderem Maße gefordert, wenn der Zufall nicht mehr gelenkter, kalkulierter Moment und Teil der Inszenierung ist, sondern tatsächlich als Realität in eine Show hereinbricht. In gewisser Weise ist das in jeder Live-Show der Fall, in Form von Fehlern und Pannen: Die Saite reißt, der Monitor-Sound ist schlecht, ein Bühnen-Licht fällt aus, dem Sänger ist der Text entfallen etc. Diese Pannen werden, wenn sie professionell gehandhabt, also z.B. rasch behoben oder souverän überspielt, werden, vom Publikum häufig kaum

8 Auch in den Diskursen zur Erforschung von populärer Musik lassen sich übrigens Spuren von Virtuosität und Dilettantismus finden: Der Pop-sensible Literaturwissenschaftler Eckhard Schumacher (2010: 210) spricht in seinen Überlegungen zum Besser- und Fan-Wissen im Pop-Diskurs in Anlehnung an Diedrich Diederichsen von dessen Wunsch nach weniger disziplinärer Professionalisierung als vielmehr professionalisiertem Dilettantismus für den wissenschaftlichen Pop-Diskurs. 
wahrgenommen, weil der Ablauf der Inszenierung dadurch nicht wirklich gestört wird. Anders ist es, wenn der Zufall die Aufführung determiniert. Dann liegt die Aufmerksamkeit der Zuschauenden ganz auf der Frage, wie die Künstlerinnen und Künstler mit dem Unvorhergesehenen umgehen, denn hier verspricht die Inszenierung durchlässig zu werden für einen Blick >hinter die Kulisse<. Darum ist hier vom Star neben der künstlerisch-performativen Arbeit ein besonderes Maß an Selbst-Darstellung gefordert - was ihm Gelegenheit bietet, über das geprobte Material hinaus etwas Außerordentliches zu zeigen, sich gewissermaßen im Wettbewerb mit sich selbst zu steigern und schließlich durch Virtuosität zu glänzen.

Wie diese situativ-performative Virtuosität ihre Wirkung entfaltet, lässt sich zeigen am Beispiel eines Open Air-Konzerts des Schlager-Stars Helene Fischer im Olympiastadion in Berlin am 5. Juli 2015, das wegen eines schweren Gewitters abgebrochen werden musste. ${ }^{9}$ Das Konzert ist Teil der Tour zum Album Farbenspiel im Jahr 2015, eine ausgesprochen aufwändige und sehr genau geplante Show: 17 Musiker, 12 Tänzerinnen und Tänzer, sechs Kostümwechsel, akrobatische Flugshow - wahrscheinlich ist Fischer derzeit der einzige deutsche Star, der eine solch aufwändige Form des Live-Entertainments zeigt, die eher für US-amerikanischen Topstars wie Beyoncé typisch ist. ${ }^{10}$ Darüber hinaus hat man auf der Fischer-Tour das Repertoire der Publikums-Beteiligung um eine Smartphone-App erweitert, die es den Besitzern bei einem bestimmten Song erlaubt, selbst mit ihren Geräten die Light Show zu generieren. Die App übersetzt die Musik in Helligkeit und Farbigkeit des Displays, im Konzert erscheint der Publikumsraum als respondierendes Element der Musik und als Live-Transformation des Tour-Titels Farbenspiel

9 http://www.bild.de/unterhaltung/musik/helene-fischer/konzertabbruch-wege n-unwetter-41655006.bild.html (Stand vom 17.2.2017);

http://www.abendblatt.de/vermischtes/article205446727/Helene-Fischer-kapi tuliert-vor-dem-Unwetter-und-bricht-ab.html (Stand vom 17.2.2017);

http: / / www.morgenpost.de/berlin/article205446011/Helene-Fischers-Konzertin-Berlin-wegen-Unwetter-abgebrochen.html (Stand vom 17.2.2017).

10 Dies zeigt sich in dem enormen finanziellen, technischen und inszenatorischen Aufwand der Show: "Der technische Aufwand für die Fischer-Show ist immens, die Vorbereitungen für die Tournee dauerten 18 Monate. 250 Beteiligte sind rund sechs Wochen lang durch Europa unterwegs, um 22 Konzerte in 14 Stadien auf die Bühne zu bringen. Der Tross von 41 Trucks und sieben Nightlinern wird bis zum Ende der Tournee mehr als 8000 Kilometer zurückgelegt haben. Die Bühne ist 52 Meter breit, 26 Meter tief und 18 Meter hoch und wiegt 52 Tonnen. Videowände links und rechts der Bühne tragen Helene Fischer bis in die letzte Reihe. Für die Technik am Abend werden Stromanschlüsse von fast 4000 Ampere benötigt. Das sind Anschlusswerte, die normalerweise für 2500 Einfamilienhäuser benötigt werden « (HA/dpa, zit. n. Hamburger Abendblatt vom 6 . Juli 2015, http://www.abendblatt.de/vermischtes/article205446727/Helene-Fi scher-kapituliert-vor-dem-Unwetter-und-bricht-ab.html (Stand vom 17.2.2015). 
(vgl. Flath 2016). Die Fischer-Show adressiert hier modern und integrativ anders als das Stereotyp vom Schlager als Musik alter, provinzieller Modernisierungsverlierer es will - ein junges, technikaffines Publikum, das sich an einem schnelle, urbanen Lifestyle orientiert (den auch der Titel »Atemlos« in seinen Lyrics trifft; vgl. Siebert 2014).

In diese Inszenierung, die sogar die private Technikausstattung der Zuschauer einkalkuliert, bricht am 5. Juli 2015 der Zufall ein - in Form eines schweren Gewitters, das um 22:30 zum Abbruch des Konzerts zwingt. Auf der Tour wird der größte Hit, »Atemlos«, erst als Zugabe gespielt und da es unter diesen Umständen keine Zugaben geben kann, zieht man das Lied als letztes Lied des Abends vor. Diverse Videoaufnahmen dieses verfrühten Konzert-Endes sind auf YouTube $\mathrm{zu}$ sehen ${ }^{11}$ und an ihnen lassen sich exemplarische Beobachtungen über das Verhältnis von Planung und Zufall und darin entstehende Virtuositäten machen. Unser Blick richtet sich insbesondere auf die inszenatorische Rahmung und den situativ-performativen Umgang mit dem Regen als hereinbrechender Umwelt; dafür nehmen wir eine kulturwissenschaftlich-hermeneutische Perspektive ein, die theaterund medienwissenschaftlich geprägt ist.

Auch wenn wir an dieser Stelle die Seite der Show-Produktion und ihre Logiken nicht in den Blick nehmen, lässt sich doch postulieren, dass es heute vermutlich keinen Veranstalter mehr gibt, der für ein Open Air-Ereignis dieser Größenordnung keine genauen meteorologischen Prognosen hat. Dass ein Gewitter droht, dürfte man schon am Nachmittag gewusst haben, als Profis werden sich alle Beteiligten darauf vorbereitet haben. Insofern steckt in der Bewältigung dieses Zufalls eine gute Portion Planung. Allerdings ist weder kalkulierbar, wie schwer und lang genau ein Sommergewitter letztlich sein wird, noch ist es möglich, Teile der Show völlig neu zu entwerfen. Tatsächlich kann man im Vergleich der Video-Mitschnitte mit dem Live-Mitschnitt des Stadionkonzerts für das ZDF auch sehen: Der IntroTeil des Songs »Atemlos « ist choreografisch der gleiche; Fischer bewegt sich auf dem runden Steg vor der Bühne. Sie trägt in diesem Fall allerdings nicht das Originalkostüm, statt eines weißen Shirts trägt sie ein grünes, mit Glanzperlen übersätes Shirt. Schon in den ersten Zeilen betont der Star die

11 Viele dieser Videos sind Mitschnitte, die aus dem Publikum gemacht wurden und darum eher unruhig und gelegentlich unscharf sind und häufig Publikum im Bild haben. Es existieren aber auch einige Videos, die mit vergleichsweise hoher Auflösung und ruhiger Kameraführung einen guten Blick auf das Bühnengeschehen ermöglichen. Darunter ist ein offizielles Video: https: / www.youtube. com/watch?v=YTjAqlyFsWw (Stand vom 17.2.2017) sowie zwei Videos, die das Geschehen am Schluss zeigen: https://www.dailymotion.com/video/x3ewa7u und https://www.youtube.com/watch?v=zrLso6FpuUg (Stand vom 17.2.1017). 
Einzigartigkeit des Moments: »Ja, das werde ich nie vergessen«. Damit stellt Fischer erstens Nähe zum Publikum her, weil sie Einblick in ihr Empfinden des Moments gibt. Zweitens spricht sie aus, was viele - so kann man vermuten - auch gerade denken und stärkt so kommunikativ die Verbindung zwischen sich und den Fans. Der an dieses langsame Intro anschließende Performance-Teil wird zwar gleich eingeleitet: »Seid Ihr bereit?«, verläuft dann aber gekürzt und verändert. Ein Teil der Originalchoreografie wird getanzt, vieles ist aber durch den regennassen Boden nicht möglich. So entfallen zum Beispiel alle Hebungen. Ein Glücksfall ist sicher, dass der Schluss der Choreografie nicht nur regenkompatibel ist, sondern hierdurch sogar besonderen Ausdruck gewinnt: Dass mit dem Stampfen der Tänzerinnen und Tänzer auf den Boden die Wassertropfen im Scheinwerferlicht aufspritzen und glitzern, steigert die Expressivität erheblich und unterstreicht das im Songtext artikulierte Gefühl von lebendigem Gegenwartserlebnis und unbedingter Körperlichkeit. Auch das Kostüm passt sich perfekt ein: Die glitzernden Regentropfen verschmelzen mit den Glanzpunkten auf dem grünen Shirt, so dass die Künstlerin sich optisch mit dem Regen zu verbinden scheint. ${ }^{12}$ Insbesondere in den professioneller gefilmten Video-Mitschnitten erscheint dieses Outfit wie eigens ausgewählt, um die Lust am Regen reizvoll in Szene zu setzen.

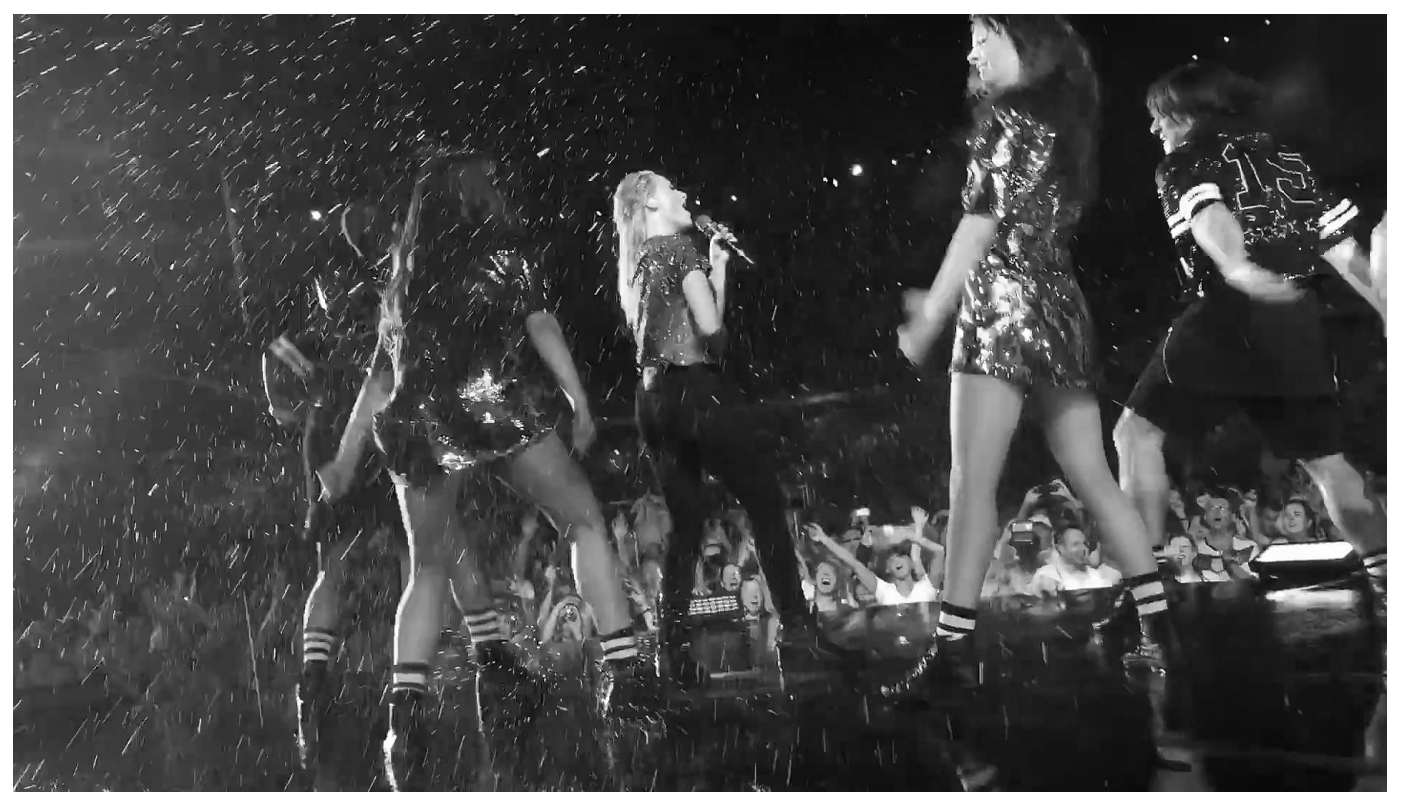

Abbildung 1: Choreografie zum Schluss von »Atemlos«. Videostill aus dem Mitschnitt https://www.youtube.com/watch?v=zrLso6FpuUg ab 00:35

12 Dass das nasse und glitzernde Shirt frappierend an das Kostüm bzw. die Erscheinung einer Meerjungfrau erinnert, ist wohl kaum kalkuliert, aber ein Effekt, der die ssaubere Sexualität der Starfigur Fischer noch hervorhebt. 
Der erste Teil folgt also weitgehend der Inszenierung, der zweite modifiziert diese, beide bleiben allerdings im Rahmen planbarer Interaktion. Erst am Schluss des Konzerts nutzt Fischer den Zufall für sich: Nach dem Abschluss der Nummer, vielen Verbeugungen in verschiedene Richtungen, während die Namen der Beteiligten über die seitlichen Screens flimmern und als die Musiker und Tänzer bereits die Bühne verlassen, verlangen die Zuschauer - in gewisser Weise der Konvention des Popkonzerts gehorchend - eine Zugabe. Und Fischer gibt eine. ${ }^{13}$ Ohne Begleitung beginnt sie einige Zeilen aus »Purple Rain « von Prince zu singen und sich dabei in eine Wasserlache zu legen. Als sie wieder aufsteht, zeigt sie ihre triefende Nässe durch das Schütteln der Haare und bekräftigt noch einmal: »Ich hab es so geliebt, im Regen mit euch.«

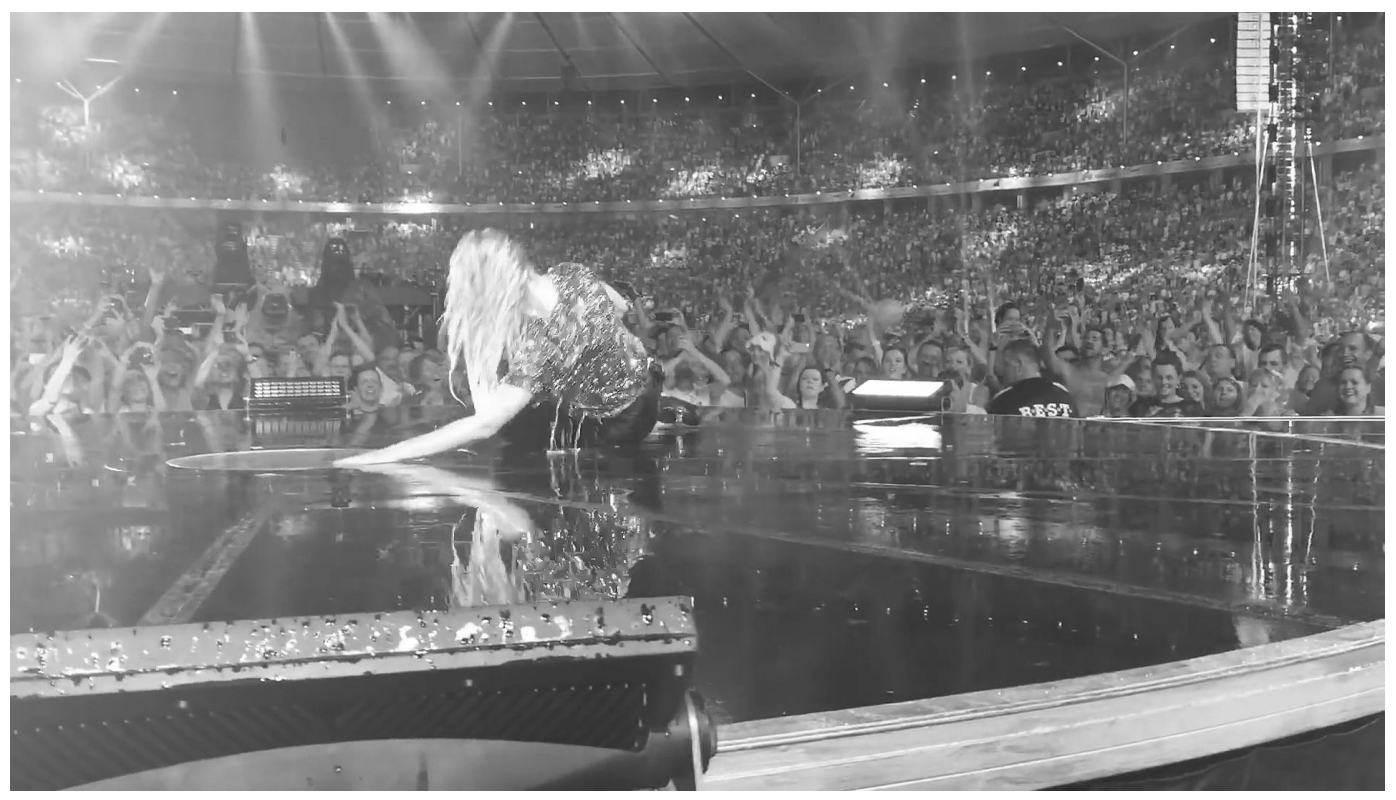

Abbildung 2: Helene Fischer performt »Purple Rain«. Videostill aus dem Mitschnitt https: / /www.youtube.com/watch?v=zrLso6FpuUg ab 03:29

Fischer gelingt es, die zufällig eingetretene Situation virtuos mehrfach zu nutzen, indem sie sowohl ihre Starfigur zeigt als auch das Gemeinschaftserlebnis forciert. Zunächst zeigt sie sich als professionelle Künstlerin, die die Show auch bei widrigen Bedingungen durchzieht. Auf der Ebene der Inszenierung passt sich Fischer - wie auch die ganze Inszenierung - nicht nur der Situation an, manche Effekte werden durch den Regen sogar verstärkt. Als sie am Ende den Prince-Klassiker »Purple Rain« - sicher nicht zufällig ein Titel, der zum Titel Farbenspiel exzellent passt - zitiert, stellt sie einen

13 Vgl. den Video-Mitschnitt https://www.dailymotion.com/video/x3ewa7u ab 3:10 bzw. https://www.youtube.com/watch?v=zrLso6FpuUg ab 3:27 (Stand vom 17.2.2017). 
Bezug zum internationalen Popgeschäft und seinen Legenden her und zeigt sich auf diese Weise als breit orientierte Entertainerin jenseits des SchlagerGenres. Sie ist in der Lage, ihr Image nicht nur performativ hervorzubringen, sondern potentiell zu erweitern:

»Although classified as a `Schlagersängerin`, Fischer has changed the image of the schlager singer progressively but radically [...]. Her stage show, in which she sings, dances and even performs stunts, displaying not only great ability as a performer but also, more unusually for schlager, great sensuality« (Mendívíl 2017: 105).

Außerdem produziert Fischer einen einzigartigen Moment, der überhaupt nur durch den Regen möglich ist, einen Moment, der die Flüchtigkeit und Einmaligkeit dieser besonderen Aufführung betont. Während das Absolvieren der Show vor allem ihre Professionalität beweist, liegt im Zitat von »Purple Rain« ein Moment von Übersteigerung, das sie als virtuose Performerin auszeichnet: Ihr Publikum hat zwar eine halbe Stunde weniger Konzert sehen können, dafür aber einen einzigartigen, unwiederholbaren Moment geteilt. Gerade weil das Publikum über Kennerschaft bezüglich der Grenzen üblicher Live-Performances verfügt, kann es diesen Moment sowohl kognitiv als emotional (vgl. Hügel 2003: 491) als virtuos begreifen und schätzen. Die geteilte Emotionalität ist wiederum für eine Konzeptionierung von Virtuosität als Steigerungs-Moment von wesentlicher Bedeutung:

»In jedem Fall sollte eine Verhandlung des Virtuosen dort ansetzen, wo die Selbstvergewisserungen einer Kulturindustrie, die sowohl die Reste bürgerlicher Kunstverehrung als auch die Massenunterhaltung bedient, heute für gewöhnlich aufhören: bei den starken Emotionen, die Performen bei Performenden und der Performance Beiwohnenden katalysiert « (Brandstetter/ Brandl-Risi/Eikels 2017: 12).

\section{Fazit}

Wir haben verschiedene Arten von Virtuosität und Zufall in popmusikalischen Performances zu identifizieren und hier an einem Fallbeispiel zu überprüfen versucht. Offenbar lassen sich in dieser zugegebenermaßen zugespitzten, ganz besonderen Performance von Helene Fischer auf bestimmten Ebenen musikalisch-technische, sozial-kommunikative und situativ-performative Virtuositäten erkennen. Zudem scheinen bei Fischers Berliner Unwetter-Auftritt die verschiedenen Umgangsformen mit Zufall im Rahmen von Pop-Inszenierungen im wahrsten Sinn des Wortes eine, ihre Rolle ge- 
spielt zu haben: Das Ereignis startete im Olympiastadion als absolut geplante Performance mit Showbusiness-Elementen, die auch das Publikum einbeziehen, antizipiert daher geplante kleinere Unkalkulierbarkeiten, lenkt Zufälle aleatorisch, ohne das Gesamtkonzept zu gefährden und wird dann vom 'wirklichen Z Zufall in Form eines Unwetters überrascht. Bei all dem ergeben sich ungeplante Räume, die hier von Fischer performativ genutzt werden.

Würde man an herkömmliche Konzepte von Virtuosität und die auch bei Heister nur in der Herleitung der Begriffe vorgebrachte Dualität von Virtuosität und Dilettantismus anschließen, ließe sich sogar sehr deutlich zeigen, inwiefern Fischer hier als virtuos beschrieben werden kann:

»Virtuosität ist, unabhängig vom Gebiet, die Hoch- bis Höchstleistung, und zwar die jeweils historisch - sozial, kulturell, nach gesellschaftlichen Sphären u.a.m. - mögliche Höchstleistung. Gegentyp der Virtuosität ist Dilettantismus, das Wenig- oder Nichtskönnen, >unprofessionelles`Vorgehen usw. [...] Virtuosität drängt nach immer weiterer Optimierung, unendlicher Perfektionierung: ein immanentes Fortschritts-Prinzip« (Heister 2004: 17).

Wobei es wegen der vielfältigen Mischformen und Multidimensionalitäten naheliegend erscheint, Virtuosität und Dilettantismus nicht bipolar, sondern als gegenüber liegende Endpunkte einer Skala zu verstehen.

Virtuosität im genannten Sinn wird hier von Fischer musikalisch-technisch, sozial-kommunikativ, vor allem aber situativ-performativ geleistet. Der >wirkliche Zufall dient also gewissermaßen als virtuelle, im Sinne von mögliche, Leerstelle, um Können zu beweisen, die Herausforderung zu meistern. Diese Leerstelle leistet für Live-Aufführungen, -Konzerte und -Performances in Pop das Gleiche, was Leerstellen generell im Star-Image leisten, z.B. das Privat-Private innerhalb des Rollenkonglomerats, Lücken oder Geheimnisse in Star-Biographien etc. Erst durch diese funktionalen Leerstellen entstehen Räume für Geschichten und aus ihnen Mythen, von denen Pop und Medien bekanntlich leben.

\section{Literatur}

Auslander, Philip (2012). »Digital Liveness. A Historico-Philosophical Perspective.« In: A Journal of Performance Art 34, Nr. 3, S. 3-11.

Brandstetter, Gabriele / Brandl-Risi, Bettina / Eikels, Kai van (2017). »Vorwort.« In: Szenen des Virtuosen (= Edition Kulturwissenschaft). Hg. v. dens. Bielefeld: transcript, S. 7-22.

Coy, Wolfgang (1999). »Berechenbares Chaos."In: Die Künste des Zufalls. Hg. v. Peter Gendolla und Thomas Kamphusmann. Frankfurt/M.: Suhrkamp, S. 34-47. 
Fischer-Lichte, Erika (2005a). »Aufführung. «In: Metzler Lexikon Theatertheorie. Hg. v. Erika Fischer-Lichte, Doris Kolesch und Matthias Warstat. Stuttgart u. Weimar: J. B. Metzler, S. 16-26.

Fischer-Lichte, Erika (2005b). »Inszenierung. «In: Metzler Lexikon Theatertheorie. Hg. v. Erika Fischer-Lichte, Doris Kolesch und Matthias Warstat. Stuttgart u. Weimar: J.B. Metzler, S. 146-153.

Flath, Beate (2016). „Co-Creation-Prozesse in Livemusikkonzerten am Beispiel der Integration von Smartphones. «In: Zeitschrift für Kulturmanagement, Nr. 2, S. 67-80.

Frith, Simon (1996). Performing Rites. On the Value of Popular Music. Cambridge: Harvard University Press.

Gendolla, Peter / Kamphusmann, Thomas (1999). »Einleitung.«In: Die Künste des Zufalls. Hg. v. Peter Gendolla und Thomas Kamphusmann. Frankfurt/M.: Suhrkamp, S. 7-14.

Großmann, Rolf (1999). »Die Phantasie der kalkulierten Welt.«In: Die Künste des Zufalls. Hg. v. Peter Gendolla und Thomas Kamphusmann. Frankfurt/M.: Suhrkamp, S. 122-136.

Grupen, Claus (1999). »Die Natur des Zufalls.«In: Die Künste des Zufalls. Hg. v. Peter Gendolla und Thomas Kamphusmann. Frankfurt/M.: Suhrkamp, S. 15-33.

Heister, Hanns-Werner (2004). »Zur Theorie der Virtuosität. Eine Skizze. «In: Musikalische Virtuosität. Hg. v. Heinz von Loesch, Ulrich Mahlert und Peter Rummenhöller. Mainz u.a.: Schott, S. 17-38.

Hornberger, Barbara (2017). »Live/Life Extensions. Über Popkonzerte als performative Dispositive. «In: Große Formen in der populären Musik. Hg. v. Christofer Jost und Knut Holtsträter. Freiburg: Waxmann (im Druck).

Hügel, Hans-Otto (2003). »Virtuose. «In: Handbuch Populäre Kultur. Begriffe, Theorien und Diskussionen. Hg. v. Hans-Otto Hügel. Stuttgart u. Weimar: J. B. Metzler, S. 491-496.

Hügel, Hans-Otto (2007). »>Weißt Du wieviel Sterne stehen? « Zu Begriff, Funktion und Geschichte des Stars. «In: Ders., Lob des Mainstream. Zu Begriff und Geschichte von Unterhaltung und Populärer Kultur. Köln: von Halem, S. 148-168.

Jacke, Christoph (2011). »Public Images Unlimited: Multimediale Gesamt-Texte. Imagekonstruktionen von Popmusik-Stars in Musikclips und darüber hinaus. «In: Imageb(u)ilder. Vergangenheit, Gegenwart und Zukunft des Videoclips. Hg. v. Thomas Mania, Henry Keazor und Thorsten Wübbena. Münster: Telos, S. 72-95.

Jacke, Christoph (2013). »Inszenierte Authentizität versus authentische Inszenierung: Ein Ordnungsversuch zum Konzept Authentizität in Medienkultur und Popmusik. «In: Ware Inszenierungen. Performance, Vermarktung und Authentizität in der populären Musik. Hg. von Dietrich Helms und Thomas Phleps (= Beiträge zur Popularmusikforschung 39). Bielefeld: transcript, S. 71-95.

Kuppens, An H. / Pol, Frank van der (2014). » True< Black Metal: The Construction of Authenticity by Dutch Black Metal Fans. «In: Communications 39, Nr. 2, S. $151-167$.

Lowry, Stephen (2003). "Star.«In: Handbuch Populäre Kultur. Begriffe, Theorien und Diskussionen. Hg. v. Hans-Otto Hügel. Stuttgart u. Weimar: J. B. Metzler, S. 441-445.

Mendívil, Julio (2017). »Rocking Granny's living room? The new voices of German schlager.«In: Perspectives on German Popular Music. Ashagte Popular Music and Folk Series. Hg. v. Michael Ahlers und Christoph Jacke. London u. New York: Routledge. S. 100-107. 
Motte-Haber, Helga de la (2004). "Schwierigkeit und Virtuosität. «In: Musikalische Virtuosität. Hg. v. Heinz von Loesch, Ulrich Mahlert und Peter Rummenhöller. Mainz u.a.: Schott, S. 175-179.

Schechner, Richard (1988). Performance Theory. New York u. London: Routledge (revised and expanded edition).

Schulze, Holger (1999). „Das Modell der nichtintentionalen Werkgenese. Über Werkgeneratoren zwischen Cage und Frontpage."In: Die Künste des Zufalls. Hg. v. Peter Gendolla und Thomas Kamphusmann. Frankfurt/M.: Suhrkamp, S. 94-121.

Schumacher, Eckhard (2010). »Existentielles Besserwissen. Dilettantismus und Professionalität im Pop-Diskurs. «In: Dilettantismus als Beruf. Hg. v. Safia Azzouni und Uwe Wirth. Berlin: Kulturverlag Kadmos, S. 201-217.

Siebert, Florian (2014.) »Pop-Literatur. Zur atemberaubenden Popularität von Helene Fischers >Atemlos durch die Nacht< (Text: Kristina Bach), vom Text ausgehend betrachtet.«In: Deutsche Lieder. Bamberger Anthologie vom 21. Juli, https: / / deutschelieder.wordpress.com/2014/07/21/helene-fischer-atemlos-dur ch-die-nacht (Stand vom 17.2.2017).

Wicke, Peter (2004). »Virtuosität als Ritual. Vom Guitar Hero zum DJ-Schamanen.« In: Musikalische Virtuosität. Hg. v. Heinz von Loesch, Ulrich Mahlert und Peter Rummenhöller. Mainz u.a.: Schott, S. 232-243.

Zurstiege, Guido (2005). Zwischen Kritik und Faszination. Was wir beobachten, wenn wir die Werbung beobachten, wie sie die Gesellschaft beobachtet. Köln: von Halem.

\begin{abstract}
In our contribution we discuss different concepts of virtuosity focusing on popular music. Based on Peter Wicke's three dimensions of virtuosity in popular music, we suggest a modification of his concept of musical-technical virtuosity, communicative-social virtuosity, and performative-situative virtuosity. Regarding these dimensions we discuss the role of coincidences and accidents in popular music live performances. These coincidences seem to open up new spaces and possibilities for creativity and virtuosity. The modes of coincidences and their management are, on the one hand, organized or controlled coincidences as often to be observed in popular music when performers or audiences seem to (re-)act spontaneously. On the other hand, one can find uncontrolled, sreal coincidences or accidents. The case study presented here is a live performance by the famous German Schlager singer Helene Fischer at an open air concert in Berlin in the summer of 2015. This show was stopped by a sudden heavy thunderstorm which led to a - in our definition - virtuosic performance by Helene Fischer managing the weather driven sudden final song of her show.
\end{abstract}

